\title{
Erratum to: Glycolytic regulation in aestivation of the sea cucumber Apostichopus japonicus: evidence from metabolite quantification and rate-limiting enzyme analyses
}

\author{
Xiaowei Xiang ${ }^{1} \cdot$ Muyan Chen $^{2} \cdot$ Changwen Wu $^{1} \cdot$ Aiyi Zhu ${ }^{1} \cdot J^{\prime n g w e n ~ Y a n g}{ }^{1} \cdot$ \\ Zhenming Lv ${ }^{1} \cdot$ Tianming Wang ${ }^{1}$
}

Published online: 22 February 2017

(C) Springer-Verlag Berlin Heidelberg 2017

\section{Erratum to: Mar Biol (2016) 163:167 \\ DOI 10.1007/s00227-016-2936-5}

In the original publication, under section heading "Results" two subsections text were incorrectly published. The correct versions are given below.

\section{Identification of partial cDNA (complete CDS)}

of $A j$-HK gene

The cDNA of hexokinase gene is $1703 \mathrm{bp}$, consisting of a 232-bp 5'-terminal untranslated region (UTR), a 130-bp
3'-UTR and a 1341-bp open reading frame (ORF) encoding 446 amino acid residues.

Identification of partial cDNA (complete CDS) of the $A j$-PK gene

The cDNA of pyruvate kinase gene is $2280 \mathrm{bp}$, consisting of a 238-bp 5'-UTR, a 437-bp 3'-UTR and a 1605 bp ORF encoding 534 amino acid residues.

The online version of the original article can be found under doi:10.1007/s00227-016-2936-5.

Tianming Wang

wtmzjuedu@163.com; wangtianming@zjou.edu.cn

1 National Engineering Research Center of Marine Facilities Aquaculture, College of Marine Sciences, Zhejiang Ocean University, Zhoushan 316022, Zhejiang, People's Republic of China

2 Fisheries College, Ocean University of China, Qingdao 266003, People's Republic of China 